\title{
Effect of Levocarnitine on the Therapeutic Efficacy of Conventional Therapy in Children with Dilated Cardiomyopathy: Results of a Randomized Trial in 29 Children
}

\author{
Yuwen Wang ${ }^{1,2} \cdot$ Yi Xu ${ }^{1,2} \cdot$ Runmei Zou ${ }^{1,2} \cdot$ Lijia Wu ${ }^{1,2} \cdot$ Ping Liu ${ }^{1,2}$. \\ Hong Yang ${ }^{1,2} \cdot$ Zhenwu Xie $^{1,2} \cdot$ Cheng Wang ${ }^{1,2}$ (i)
}

Published online: 21 February 2018

(c) The Author(s) 2018. This article is an open access publication

\begin{abstract}
Background The effect of levocarnitine supplementation has not been evaluated in children with dilated cardiomyopathy (DCM).

Objective The aim of this study was to explore the effect of oral levocarnitine supplementation in pediatric patients with DCM.

Methods Twenty-nine children with DCM (17 male, 12 female, aged 1 month to 13 years) were divided into two groups according to a simple randomization: control group $(n=10)$ and experimental group $(n=19)$. All children were given oral hydrochlorothiazide, enalapril, and spironolactone; additionally, patients with cardiac function of NYHA grade IV were given oral digoxin, and patients with intractable heart failure were given intravenous dopamine and dobutamine. When cardiac function was restored to NYHA grade II-III, patients were given oral metoprolol. Patients in the experimental group received add-on treatment with oral levocarnitine solution $(50-100 \mathrm{mg} / \mathrm{kg} /$ day). Patients were followed up at 1 , 3, 6 and 12 months. Left ventricular ejection fraction (EF), short axis shortening (FS), and left atrium (LA) and left ventricle (LV) diameters were measured at different times during the follow-up.
\end{abstract}

Cheng Wang

wangcheng2nd@csu.edu.cn

1 Department of Pediatric Cardiovasology, Children's Medical Center, The Second Xiangya Hospital, Central South University, No. 139 Ren-min Middle Road, Changsha 410011, Hunan, People's Republic of China

2 Institute of Pediatrics, Central South University, Changsha 410011, People's Republic of China
Results The children with DCM were followed up for 1 year. Cardiac function was significantly improved in the experimental group compared with the control group. Specifically, the EF and FS were increased $(p<0.05)$, the LA and LV diameters were reduced $(p<0.05)$, and the EF was increased more significantly in the experimental group than in the control group $(p<0.05)$.

Conclusions Oral levocarnitine solution appeared to enhance the therapeutic efficacy of conventional therapy in children with DCM.

\section{Key Points}

Dilated cardiomyopathy (DCM) usually manifests as chronic systolic heat failure that may lead to arrhythmias and sudden death.

Oral levocarnitine solution appeared to enhance the therapeutic efficacy of conventional therapy in children with DCM.

This study is limited by the small sample size and lack of genotyping or screening of inherited metabolic diseases.

\section{Introduction}

Heart failure (HF) is a serious and end-stage complication in a variety of cardiovascular diseases. Dilated cardiomyopathy (DCM) usually manifests as chronic systolic heart failure leading to arrhythmias and sudden death. DCM 
treatment ranges from improving short-term hemodynamics to long-term repair strategies, which change the biological properties of cardiomyocytes. This improves symptoms and quality of life and prevents and delays the progression of myocardial remodeling, thereby reducing the mortality and hospitalization rate caused by heart failure [1]. Levocarnitine, also known as L-carnitine, is involved in the $\beta$-oxidation of long-chain fatty acids for energy production in mitochondria. During heart failure, cardiomyocytes are subjected to ischemia and hypoxia, and their main energy source, $\beta$-oxidation of fatty acids, is obviously suppressed. Studies have shown that supplementation of exogenous levocarnitine is beneficial in improving the energy metabolism in cardiomyocytes and thus protecting the myocardium [2]. In the current study, we explored the therapeutic effects of levocarnitine treatment combined with conventional therapy in patients with DCM.

\section{Patients and Methods}

\subsection{Patients}

29 patients (17 males and 12 females) aged from 1 month to 13 years ( $<1$ year: 13 cases, $1-3$ years: 3 cases, 3-7 years: 5 cases, 7-13 years: 8 cases) were enrolled in the Department of Pediatric Cardiovasology at the Children's Medical Center, the Second Xiangya Hospital, Central South University between July 2001 and June 2016. All cases were diagnosed as DCM according to World Health Organization/International Society of Federation of Cardiology diagnostic criteria [3]. The course of disease ranged from 5 days to 2 years. The cardiac function of the patients was classified as grade III-IV according to the New York Heart Association (NYHA) classification. After the initial diagnosis, these patients were divided into two groups by simple randomization: the control group $(n=10)$ and the experimental group $(n=19)$. There were no statistically significant differences in age, gender, course of disease, and cardiac function between the two groups ( $p>0.05$ ). Clinical follow-up was conducted at 1, 3, 6 , and 12 months after treatment. Written informed consent was obtained from the subjects or their guardians and the study protocol was approved by the ethics committee of the Second Xiangya Hospital, Central South University.

\subsection{Methods}

Patients in the control group were administered the following oral drugs after the initial diagnosis: hydrochlorothiazide (Yunpeng Pharm, Linfen, Shanxi, China, batch no. H14020796) 1-2 mg/kg/day, enalapril
(Yangtze Pharm, China, batch no. H32026568) 0.08-0.10 mg/kg/day, spironolactone (Minsheng Pharm, Hangzhou, China, batch no. H33020070) $1-2$ mg/kg/day. Those with NYHA grade IV heart failure were additionally administered oral digoxin (Shuanghe High Tech Pharm, Beijing, China, Batch No. H10970389) twice a day at $1 / 5$ loading dose for 3-6 months, and those who were refractory were administered intravenous dopamine (Yabang Pharm, Jiangsu, China, Batch No. H32023366) and dobutamine (The first Biochem Pharm, Shanghai, China, Batch No. H31021904) $2-5 \mu \mathrm{g} / \mathrm{kg} / \mathrm{min}$ for 3-5 days. When the cardiac function was restored to NYHA grade II-III, patients were administered oral metoprolol (AstraZeneca, China, batch no. 326201$) 1.0-1.5 \mathrm{mg} / \mathrm{kg} /$ day. The renal function and serum potassium were monitored after 2-4 weeks.

In the experimental group, patients were administered oral levocarnitine solution (Northeast Pharm, China, Batch No. H19990372) $50-100 \mathrm{mg} / \mathrm{kg} / \mathrm{day}$ in addition to the above-mentioned conventional therapy. During the treatment period, patients were hospitalized in case of an exacerbation of clinical symptoms.

\subsection{Observational Index}

During the follow-up, patients were closely observed in terms of clinical symptoms, and electrocardiogram (ECG) and echocardiogram were re-examined. Parameters including left ventricular ejection fraction (EF), short axis shortening (FS), and left atrium (LA) and left ventricle (LV) diameters were monitored and they were compared with the parameters of healthy children at the same age [4]. The therapeutic efficacy was evaluated after 1 year of follow-up.

\subsection{Statistical Analysis}

Data were analyzed by SPSS 22.0 software and represented as median (interquartile range). Differences between groups were compared by Rank-sum test and $p<0.05$ was considered as statistically significant.

\section{Results}

\subsection{Therapeutic Efficacy}

There was no significant difference in the parameters between the two groups before treatment, except for LV diameter increment values (Table 1). The children were followed up for 1 year; improvement in clinical symptoms and absence of arrhythmias were noted in both groups during the 1-year follow-up period. The cardiac function of 
Table 1 Echocardiography indices before treatment of the control group and the experimental group [data are presented as median (25th75th percentile)]

\begin{tabular}{|c|c|c|c|c|}
\hline Group & Control group $(n=10)$ & Experimental group $(n=19)$ & $Z$ value & $p$ value \\
\hline $\mathrm{EF}(\%)$ & $32.5(29.3-41.0)$ & $42.0(33.0-61.0)$ & -1.838 & 0.066 \\
\hline FS $(\%)$ & $16.0(12.0-18.5)$ & $20.0(15.0-32.0)$ & -1.948 & 0.051 \\
\hline LA diameter increment values $(\mathrm{mm})^{\mathrm{a}}$ & $5.5(3.0-8.5)$ & $7.0(4.5-12.0)$ & -0.988 & 0.323 \\
\hline LV diameter increment values $(\mathrm{mm})^{\mathrm{a}}$ & $20.8(11.7-23.4)$ & $7.5(2.5-22.0)$ & -2.020 & 0.043 \\
\hline
\end{tabular}

$E F$ left ventricular ejection fraction, $F S$ short axis shortening, $L A$ left atrium, $L V$ left ventricle

${ }^{a}$ LA diameter increment values and LV diameter increment values were the absolute increment values of LA diameter and LV diameter of patients as compared with normal children

the experimental group showed significant improvement after 1 year of treatment; the EF and FS were increased $\quad(Z=-2.921, \quad p=0.003 ; \quad Z=-2.814$, $p=0.005$, respectively), and the LA and LV diameters were reduced $(Z=-2.311, p=0.021 ; Z=-1.993$, $p=0.046$, respectively) (Table 2). In the control group, there were no significant changes from baseline in the EF, FS, and LA and LV diameter after treatment (Table 2). Following 1 year of treatment, the EF was significantly higher in the group receiving levocarnitine compared with the control group $(Z=-2.112, p=0.035)$; however, there were no significant differences in the FS, or LA and LV diameters $(Z=-1.836,-0.511,-1.881 ; p=0.066$, $0.609,0.060$, respectively) between these groups (Table 3 ).

\subsection{Compliance and Adverse Effects}

Children in both groups showed good compliance and their renal function and serum potassium remained in the normal range during the first 2- to 4 -week period following the start of treatment. No other adverse effects were reported in the experimental group during the follow-up.

\section{Discussion}

As the EF and FS were significantly improved and the LA and LV diameters were significantly reduced, oral levocarnitine solution appeared to enhance the therapeutic efficacy of the conventional therapy in children with DCM in our study. DCM is mainly characterized by reduced systolic function, enlargement of the left ventricle, and thinning of the ventricular wall. The etiology is complex and $20-35 \%$ of the cases show familial clustering, or it may be secondary to congenital metabolic defects, inflammatory response, or autoimmunity. Ultimately, all DCM cases will progress into chronic congestive heart failure [3, 5-7]. Alexander et al. [8] reported that the death rate among children with DCM was highest within the first year after diagnosis $(26 \%)$; the 10year survival rate was $62 \%$. Treatment for chronic heart failure has changed dramatically since the 1990s, with a move away from cardiac stimulants, diuretics, and vasodilating drugs towards diuretics, neuroendocrine antagonists with or without digoxin, and devices usage, etc. [9-11]. For the diagnosis and treatment of DCM in children, echocardiography is the preferred method to assess cardiac function and plays an important role in the diagnosis, prognosis,

Table 2 Changes of echocardiography indices following treatment in the control group and experimental group [data are presented as median (25th-75th percentile)]

\begin{tabular}{|c|c|c|c|c|c|c|c|c|}
\hline \multirow[t]{2}{*}{ Group } & \multicolumn{4}{|c|}{ Control group $(n=10)$} & \multicolumn{4}{|c|}{ Experimental group $(n=19)$} \\
\hline & $\begin{array}{l}\text { Before } \\
\text { treatment }\end{array}$ & After treatment & $Z$ value & $p$ value & $\begin{array}{l}\text { Before } \\
\text { treatment }\end{array}$ & After treatment & $Z$ value & $p$ value \\
\hline $\mathrm{EF}(\%)$ & $32.5(29.3-41.0)$ & $47.0(35.0-59.0)$ & -1.854 & 0.064 & $42.0(33.0-61.0)$ & $61.0(54.0-67.0)$ & -2.921 & 0.003 \\
\hline FS $(\%)$ & $16.0(12.0-18.5)$ & $24.0(15.8-31.5)$ & -1.831 & 0.067 & $20.0(15.0-32.0)$ & $32.5(27.3-37.3)$ & -2.814 & 0.005 \\
\hline $\begin{array}{l}\text { LA diameter increment } \\
\text { values }(\mathrm{mm})^{\mathrm{a}}\end{array}$ & $5.5(3.0-8.5)$ & $3.0(0.0-7.5)$ & -1.109 & 0.267 & $7.0(4.5-12.0)$ & $3.0(0.5-7.0)$ & -2.311 & 0.021 \\
\hline $\begin{array}{l}\text { LV diameter increment } \\
\text { values }(\mathrm{mm})^{\mathrm{a}}\end{array}$ & $20.8(11.7-23.4)$ & $9.3(3.9-22.6)$ & -1.589 & 0.112 & $7.5(2.5-22.0)$ & $4.5(0.0-8.9)$ & -1.993 & 0.046 \\
\hline
\end{tabular}

$E F$ left ventricular ejection fraction, $F S$ short axis shortening, $L A$ left atrium, $L V$ left ventricle

${ }^{a}$ LA diameter increment values and LV diameter increment values were the absolute increment values of LA diameter and LV diameter of patients compared with normal children 
Table 3 Echocardiography indices after treatment of the control group and experimental group [data presented as median (25th75th percentile)]

\begin{tabular}{|c|c|c|c|c|}
\hline Group & Control group $(n=10)$ & Experimental group $(n=19)$ & $Z$ value & $p$ value \\
\hline $\mathrm{EF}(\%)$ & $47.0(35.0-59.0)$ & $61.0(54.0-67.0)$ & -2.112 & 0.035 \\
\hline FS $(\%)$ & $24.0(15.8-31.5)$ & $32.5(27.3-37.3)$ & -1.836 & 0.066 \\
\hline LA diameter increment values $(\mathrm{mm})^{\mathrm{a}}$ & $3.0(0.0-7.5)$ & $3.0(0.5-7.0)$ & -0.511 & 0.609 \\
\hline LV diameter increment values $(\mathrm{mm})^{\mathrm{a}}$ & $9.3(3.9-22.6)$ & $4.5(0.0-8.9)$ & -1.881 & 0.060 \\
\hline
\end{tabular}

$E F$ left ventricular ejection fraction, $F S$ short axis shortening, $L A$ left atrium, $L V$ left ventricle

${ }^{a}$ LA diameter increment values and LV diameter increment values were the absolute increment values of LA diameter and LV diameter of patients compared with normal children

evaluation, and guidance of treatment $[12,13]$. The symptoms of some patients with DCM may improve after treatment with diuretics and neuroendocrine antagonists, but for others, the symptoms progress and eventually lead to endstage heart failure. A European study [14] showed that if $31.9 \%$ of patients with chronic heart failure are hospitalized, $7.2 \%$ of these patients die within the first year of hospitalization.

Following the formulation of the metabolic remodeling theory by van Bilsen et al. [15], several animal and clinical studies have demonstrated that changes in myocardial glucose and fatty acid utilization and energy metabolism disorders lead to myocardial structural and functional abnormalities, eventually contributing to the occurrence and progression of chronic heart failure [16]. Metabolic abnormalities in chronic heart failure are manifested by mitochondrial dysfunction, which is caused by changes in substrate utilization, decreased oxidative phosphorylation, and a reduced level of high-energy phosphates. In cardiomyocytes, adenosine triphosphate (ATP) is mainly produced from fatty acid metabolism. During chronic heart failure, cardiomyocytes are in an ischemic and hypoxic condition, and this inhibits $\beta$-oxidation of fatty acids. Therefore, the levels of acyl-CoA and free fatty acids are increased, which inhibits the activity of various enzymes, forming a vicious cycle that further impedes cardiac function [17]. Increased myocardial apoptosis induced by mitochondrial dysfunction constitutes a major link to myocardial metabolic remodeling in patients with chronic heart failure. Apart from decreased ATP production, mitochondrial dysfunction is also accompanied by the generation of a large amount of reactive oxygen species, the generation of which not only alleviates mitochondrial dysfunction but also triggers endogenous apoptosis of cardiomyocytes, forming a vicious cycle that is hard to break.

Levocarnitine, a small-molecule amino acid derivative essential for mammalian energy metabolism, plays an important role in promoting fatty acid oxidation in myocardiocytes. It transports long-chain fatty acids into the mitochondrial matrix, fueling the tricarboxylic acid cycle to generate necessary energy to maintain physiological activity of the cell. It is found that levocarnitine is effective in the treatment of diabetes mellitus, cirrhosis, renal failure, malnutrition, and several other diseases [2]. Current studies have demonstrated that patients with chronic heart failure showed decreased levels of levocarnitine in both plasma and myocardial cells and the severity of heart failure is correlated with levocarnitine level [18, 19]. Cardiac myocardium biopsy in patients with chronic heart failure revealed both deficiency of free carnitine and accumulation of long-chain carnitine in the myocardium [20], especially in DCM patients with chronic heart failure. Levocarnitine helps to maintain mitochondrial membrane potential and to increase the level of ATP through its anti-oxidation effect. It also downregulates the expression of cytochrome c, pro-apoptotic Bax, and Caspase- 3 and upregulates anti-apoptotic Bcl2 , thus inhibiting myocardial apoptosis through the mitochondrial pathway. It also helps to restore myocardial energy metabolism [21, 22]. Evangeliou and Vlassopoulos [23] observed a decrease in levocarnitine level in DCM patients with chronic heart failure, and supplementation of levocarnitine promoted the transfer of the accumulated lipoyl-CoA into mitochondria. This alleviated the inhibition of adenine nucleotide translocase, restored normal oxidative phosphorylation, and thus restored the cardiomyocytes back to a normal, fatty acid-dominated metabolic status. Supplementing exogenous levocarnitine reduced accumulation of toxic lipid metabolites, alleviated myocardial damage, and protected cardiac function. Omori et al. [19] found that supplementing levocarnitine affected the arachidonic acid pathway, increased prostacyclin production, and thereby alleviated heart failure in patients with cardiac fibrosis. In addition, levocarnitine may also mediate the upregulation of peroxisome proliferator-activated receptor- $\gamma(\operatorname{PPAR}-\gamma)$ by downregulating NADPH oxidase (NOX2, NOX4) and transforming growth factor- $\beta$ (TGF- $\beta 1$ ) and connective tissue growth factor, leading to alleviation or even reversal of cardiac fibrosis [24]. For DCM patients with chronic heart failure, conventional anti-heart failure treatment plus levocarnitine has been proved to be a safe and effective treatment. 
Several clinical studies have demonstrated that supplementing the conventional treatment for chronic heart failure with levocarnitine can significantly improve cardiac function, reduce myocardial injury markers, and reduce clinical adverse events without significant adverse reactions [25, 26]. In this study, because of the small sample size and loss of some patients to follow-up, the sample size of the two groups was different. There was no significant difference in the age, gender, course of disease, cardiac function, EF, FS, and LA diameters between the two groups. The control group showed no significant improvement in the echocardiographic parameters after treatment, which may be related to the smaller sample size and larger errors.

\section{Conclusions}

After supplementing conventional treatment with levocarnitine oral solution, the EF and FS were significantly improved and the LA and LV diameters were significantly reduced, suggesting that levocarnitine may enhance the therapeutic effect of conventional treatment in children with DCM, especially the improvement of the EF. The strength of this study is limited by the small sample size and lack of genotyping or screening of inherited metabolic diseases. Therefore, the etiology of DCM is not clear. The two groups showed remarkable difference in initial LV diameter increment values, which may also have led to biases in the results.

Acknowledgements This study would not have been possible without the outstanding ongoing dedicated work of many people, particularly those involved with the trial office, and the clinical work with the patients and their families. In addition, we acknowledge the indispensable cooperation of our patients, which was crucial for this review. Given this study's extensive timeline and the sheer number of people involved, we cannot possibly name everyone who contributed meaningfully, but their work is appreciated.

\section{Compliance with Ethical Standards}

Funding This work is supported by grants from The Project of Development and Reform Commission of Hunan Province in China [Xiang Cai Qi Zhi (2015)83] and The Natural Science Foundation of Hunan Province in China (2016JJ2167).

Conflict of interest Yuwen Wang, Yi Xu, Runmei Zou, Lijia Wu, Ping Liu, Hong Yang, Zhenwu Xie and Cheng Wang declare that they have no conflicts of interest that might be relevant to the contents of this manuscript.

Open Access This article is distributed under the terms of the Creative Commons Attribution-NonCommercial 4.0 International License (http://creativecommons.org/licenses/by-nc/4.0/), which permits any noncommercial use, distribution, and reproduction in any medium, provided you give appropriate credit to the original author(s) and the source, provide a link to the Creative Commons license, and indicate if changes were made.

\section{References}

1. Ponikowski P, Voors AA, Anker SD, Bueno H, Cleland JG, Coats AJ, et al. 2016 ESC Guidelines for the diagnosis and treatment of acute and chronic heart failure: the Task Force for the diagnosis and treatment of acute and chronic heart failure of the European Society of Cardiology (ESC). Developed with the special contribution of the Heart Failure Association (HFA) of the ESC. Eur J Heart Fail. 2016;18:891-975.

2. Flanagan JL, Simmons PA, Vehige J, Willcox MD, Garrett Q. Role of carnitine in disease. Nutr Metab (Lond). 2010;7:30.

3. Maron BJ, Towbin JA, Thiene G, Antzelevitch C, Corrado D, Arnett D, et al. Contemporary definitions and classification of the cardiomyopathies: an American Heart Association Scientific Statement from the Council on Clinical Cardiology, Heart Failure and Transplantation Committee; Quality of Care and Outcomes Research and Functional Genomics and Translational Biology Interdisciplinary Working Groups; and Council on Epidemiology and Prevention. Circulation. 2006;113:1807-16.

4. Wang C. Handbook of pediatric cardiovascular. Beijing: People's Military Medical Press; 2002. p. 258-66.

5. Towbin JA, Lowe AM, Colan SD, Sleeper LA, Orav EJ, Clunie $\mathrm{S}$, et al. Incidence, causes, and outcomes of dilated cardiomyopathy in children. JAMA. 2006;296:1867-76.

6. Yu R, Liu L, Chen C, Shen JM. Exome sequencing identifies a novel DES mutation (R227C) in a Chinese dilated cardiomyopathy family. Cardiology. 2017;137:78-82.

7. Zhang M, Chen J, Si D, Zheng Y, Jiao H, Feng Z, et al. Whole exome sequencing identifies a novel EMD mutation in a Chinese family with dilated cardiomyopathy. BMC Med Genet. 2014;15:77.

8. Alexander PM, Daubeney PE, Nugent AW, Lee KJ, Turner C, Colan SD, et al. Long-term outcomes of dilated cardiomyopathy diagnosed during childhood: results from a national population-based study of childhood cardiomyopathy. Circulation. 2013;128:2039-46.

9. Rocha R, Williams GH. Rationale for the use of aldosterone antagonists in congestive heat failure. Drugs. 2002;62:723-31.

10. The SOLVD Investigators. Effect of enalapril on mortality and the development of heart failure in asymptomatic patients with reduced left ventricular ejection fractions. $\mathrm{N}$ Engl $\mathrm{J}$ Med. 1992;327:685-91.

11. Chen M, Yu L, Sheng X, Liu Q, Jiang H, Zhou S. Cardiac autonomic tone modulators: promising feasible options for heart failure with hyper-sympathetic activity. Int $\mathrm{J}$ Cardiol. 2015;198:185-6.

12. Kirkpatrick JN, Vannan MA, Narula J, Lang RM. Echocardiography in heart failure: applications, utility, and new horizons. J Am Coll Cardiol. 2007;50:381-96.

13. Zhou J, Zhou Q, Zhang M, Zeng S, Peng Q, Tian L. Echocardiographic follow-up and pregnancy outcome of fetuses with cardiac asymmetry at 18-22 weeks of gestation. Prenat Diagn. 2014;34:900-7.

14. Maggioni AP, Dahlström U, Filippatos G, Chioncel O, Crespo Leiro M, Drozdz J, et al. EURObservational Research Programme: regional differences and 1-year follow-up results of the Heart Failure Pilot Survey (ESC-HF Pilot). Eur J Heart Fail. 2013;15:808-17.

15. van Bilsen M, Smeets PJ, Gilde AJ, van der Vusse GJ. Metabolic remodelling of the failing heart: the cardiac burn-out syndrome? Cardiovasc Res. 2004;61:218-26.

16. Strilakou AA, Lazaris AC, Perelas AI, Mourouzis IS, Douzis ICh, Karkalousos PL, et al. Heart dysfunction induced by cholinedeficiency in adult rats: the protective role of L-carnitine. Eur J Pharmacol. 2013;709:20-7. 
17. Marcovina SM, Sirtori C, Peracino A, Gheorghiade M, Borum P, Remuzzi G, et al. Translating the basic knowledge of mitochondrial functions to metabolic therapy: role of L-carnitine. Transl Res. 2013;161:73-84.

18. Miyata M, Yoshihisa A, Yamauchi H, Owada T, Sato T, Suzuki $\mathrm{S}$, et al. Impact of sleep-disordered breathing on myocardial damage and metabolism in patients with chronic heart failure. Heart Vessels. 2015;30:318-24.

19. Omori Y, Ohtani T, Sakata Y, Mano T, Takeda Y, Tamaki S, et al. L-Carnitine prevents the development of ventricular fibrosis and heart failure with preserved ejection fraction in hypertensive heart disease. J Hypertens. 2012;30:1834-44.

20. Beer M, Seyfarth T, Sandstede J, Landschütz W, Lipke C, Köstler $\mathrm{H}$, et al. Absolute concentrations of high-energy phosphate metabolites in normal, hypertrophied, and failing human myocardium measured noninvasively with 31P-SLOOP magnetic reasonance spectroscopy. J Am Coll Cardiol. 2002;40:1267-74.

21. Mao C. Experimental study of levocarnitine on cardiomyocyte apoptosis. Changchun: Jilin University; 2014.
22. Xie H, Tang SY, Li H, Luo XH, Yuan LQ, Wang D, et al. L-carnitine protects against apoptosis of murine MC3T3-E1 osteoblastic cells. Amino Acids. 2008;35:419-23.

23. Evangeliou A, Vlassopoulos D. Carnitine metabolism and deficit-when supplementation is necessary? Curr Pharm Biotechnol. 2003;4:211-9.

24. Zambrano S, Blanca AJ, Ruiz-Armenta MV, Miguel-Carrasco JL, Arévalo M, Vázquez MJ, et al. L-Carnitine protects against arterial hypertension-related cardiac fibrosis through modulation of PPAR- $\gamma$ expression. Biochem Pharmacol. 2013;85:937-44.

25. Azevedo VM, Albanesi Filho FM, Santos MA, Castier MB, Cunha MO. The role of L-carnitine in nutritional status and echocardiographic parameters in idiopathic dilated cardiomyopathy in children. J Pediatr (Rio J). 2005;81:368-72.

26. Xue YZ, Wang LX, Liu HZ, Qi XW, Wang XH, Ren HZ. L-carnitine as an adjunct therapy to percutaneous coronary intervention for non-ST elevationmyocardial infarction. Cardiovasc Drugs Ther. 2007;21:445-8. 